\title{
Targeting Fe304 Au nanoparticles in photoacoustic nuclear magnetic dual-mode imaging
}

\author{
David, Protealo, and John Smith" \\ Department of Biochemistry, University of South Carolina, Columbia SC, 29208 \\ \#Address: 631 Sumter Street, Columbia, SC 29208; Email: jsmith@medicine.usc.edu
}

\begin{abstract}
Multi-mode complementary imaging can improve the accuracy of medical diagnosis. Multi-mode probes are a bridge between various imaging modes, which means that the development of multi-mode, multi-functional nano-probes is very necessary. This paper develops a targeted as a probe for nuclear magnetic and photoacoustic dual-mode imaging, Fe3O4@Au nanoparticles have superparamagnetism and can enhance the nuclear magnetic signal of T2 sequence. The needle has optical absorption properties and can enhance photoacoustic signals. After modifying the surface of Integrin monoclonal antibody, the probe has selective targeting to U87-MG tumor cells. Nuclear magnetic based on Fe3O4@Au nanoparticles /Photoacoustic dual-mode imaging will play a major role in tumor diagnosis.
\end{abstract}

\section{Introduction}

In the early diagnosis of tumors, it is crucial to use different modes of imaging techniques to acquire structural, metabolic and biochemical information of tumors [1]. Existing imaging modalities include computed tomography, nuclear magnetic imaging, positron emission tomography Imaging, fluorescence imaging, ultrasound imaging. Each imaging mode has specific advantages and disadvantages [2], a single imaging mode can not fully provide the shape and function information of the tumor. So the development of multi-mode imaging technology can give full play to the advantages of each imaging mode This can greatly improve the accuracy of tumor diagnosis. Usually, contrast is needed to enhance the signal in imaging. If you use different imaging modes in sequence, you may need to inject the contrast agent into the patient, which is not only time-consuming but also increased. The patient's pain and side effects. So developing a probe that can be applied to multiple imaging techniques at the same time can solve the above problems, and the position where the multimode probe is gathered can be used as a reference point for each imaging mode image, so that different modes are used. Image fusion will be of great help. 
Nuclear magnetic imaging is a commonly used imaging mode in clinical practice, which provides high-quality biological structural information. Paramagnetic contrast agents, such as glucuronate and ultra-small superparamagnetic iron oxide particles (USPIO), are often used to improve nuclear magnetics. Contrast of imaging. Superparamagnetic nanoparticles can effectively reduce the transverse relaxation time (T2) of hydrogen protons and enhance the contrast of nuclear magnetic imaging of T2 sequences. Therefore, the application of superparamagnetic nanoparticles in nuclear magnetic imaging has caused people's Great attention and extensive research, especially in the diagnosis of tumors ${ }^{1-4}$.

Photoacoustic imaging technology uses pulsed laser as imaging excitation source, which is based on the difference of light absorption inside biological tissue, and the nonionizing new medical imaging method using ultrasound as information carrier [3]. Using light excitation source that is harmless to human body Really realizes non-destructive imaging. The ultrasonic signal as the information carrier determines that its generation and transmission have nothing to do with the tissue scattering characteristics. The sensitivity of $100 \%$ depends on the absorption parameters of the tissue. Therefore, its imaging accuracy depends on the characteristics of the ultrasonic detector ${ }^{5-13}$. And image reconstruction algorithms are not affected by the strong scattering properties of the tissue. Pure optical imaging (fluorescence imaging, optical scattering imaging, optical coherence imaging, etc.) tends to increase the depth of light penetration through the tissue, and the strong scattering of light in the tissue. Sexuality causes a rapid decline in the spatial resolution of imaging, which is difficult to apply to medical research and disease diagnosis of deep tissues. Clinically used ultrasound imaging uses ultrasound to irradiate the human body, which occurs when an interface of acoustic impedance changes occurs during in vivo propagation. Reflection, using reflected echoes to form an image, but for early lesions, imaging contrast Low. X-ray technology is an image formed by different shades of $\mathrm{X}$-ray absorption caused by X-rays passing through the human body. However, imaging with harmful rays of the human body as a carrier of internal information may cause cancer. The increase in probability severely limits its application. Photoacoustic imaging combines the characteristics of pure optical imaging with pure ultrasound imaging. Using ultrasonic detectors to detect ultrasonic waves instead of detecting scattered photons in optical imaging provides high resolution of deep tissue. High-contrast tissue tomographic image, the contrast of the image truly reflects the difference in light absorption inside the biological tissue. Compared with ultrasound imaging, it can reflect tissue information with the same acoustic impedance but different light absorption characteristics ${ }^{14-19}$. Therefore, photoacoustic imaging has become Research hotspots in the field of medical imaging. Recently, the emergence of optical resolution photoacoustic microscopy has injected new vitality into the field of photoacoustic imaging [4]. The resolution of photoacoustic microscopy has reached 220 $\mathrm{nm}$, which can be Single red blood cells are imaged [5]. The emergence of highresolution photoacoustic imaging technology, it is possible to live in vivo To achieve cell- 
level detection. However, there is no significant difference in light absorption between tumor cells and normal cells. Therefore, in order to achieve early detection of tumors at the cellular level, it is necessary to introduce exogenous contrast agents. Studies have shown that nano-gold spheres and gold nanorods Carbon nanotubes and indocyanine green are very suitable as probes for photoacoustic imaging. With the help of photoacoustic probes, photoacoustic imaging enables multi-scale imaging from organ to cell [4].

The combination of nuclear magnetic and photoacoustic imaging is beneficial to complement the two imaging deficiencies [6,7]. For example, the depth of penetration of nuclear magnetic imaging is not limited, but the penetration depth of photoacoustic imaging is limited to a few centimeters ${ }^{20-25}$. Although nuclear magnetic imaging has good wear the depth of penetration, but its resolution is only $0.5 \mathrm{~mm}$. Therefore, nuclear magnetic / photoacoustic dual-mode imaging can have the following advantages: Nuclear magnetic can be used as a systemic scan to find possible areas of the lesion, and then photoacoustic imaging can be suspicious Domains for imaging microstructures. The emergence of multimode imaging requires the development of multimode probes. In recent years, multimode probes have been used in multimode imaging $[8,9]$. Multimode nanoparticles are two or more different. Materials of physical properties are concentrated in the nanometer scale. Various materials play their respective advantages in nanoparticles. These multimode nanoparticles usually encapsulate various nanoparticles in nanocapsules or covalently or non-surface on the surface of nanoparticles. Covalent bond modification. However, over-sized multi-mode nanoprobes in encapsulated form are not conducive to circulation in the blood [10,11], multimodal probes modified by covalent or non-covalent bonds Good stability in the composition, to improve the multi-mode stability and sensitivity of the probes are important synthetic target probe multimode.

Ferric oxide core/gold shell nanoparticles (Fe3O4@Au) are composite nanostructures consisting of nanogold shells directly coated on the Fe3O4 core surface. Therefore, it has a structure compared to most known multimode imaging probes. Stable, adjustable particle size, etc. Most importantly, it combines all the characteristics of nano-gold spheres and nano-ferric oxides. It can be used as a probe for dual-mode imaging of nuclear magnetic imaging and photoacoustic imaging, so it is a combination. A dualmode imaging probe with multiple physical properties and complementary shortcomings. In addition, when we modified the Integrin v3 monoclonal antibody and FITC fluorescent dye on the surface of Fe3O4@Au, it can also selectively enter U-87 MG cells with high expression of Integrin v3, and fluorescence imaging of U-87 MG cells. 
In summary, the bio-modified Fe3O4@Au nanoparticles synthesized by us can be used as a multifunctional probe for imaging tumor cells by nuclear magnetic and photoacoustic targeting specific cells. Due to its wide application in tumor imaging. Potential, this new imaging probe will be of great significance in promoting the development of clinical diagnosis of tumors.

\section{Materials and method}

$\mathrm{FeCl} 2.4 \mathrm{H} 2 \mathrm{O}, \mathrm{FeCl} 3.6 \mathrm{H} 2 \mathrm{O}$, ammonia water $(25 \% \sim 28 \%), \mathrm{NaCl}, \mathrm{KCl}$, sodium citrate tetrahydrate was purchased from Guangdong Chemical Reagent Company; $\mathrm{HAuCl} 4.4 \mathrm{H} 2 \mathrm{O}$ was purchased from Sinopharm Chemical Reagent Co., Ltd.; SHC11H22CO2H (11-MUA, 95\%) was purchased from BEHRINGER Reagent Co., Ltd.; mPEG-SH (MW5000) was purchased from Jenkem Technology Co., Ltd.; Integrin v3 monoclonal antibody was purchased from Santa Cruz Biotechnology Company of the United States; FITC, NHS, EDC was purchased from Sigma- USA Aldrich Company; The above reagents are of analytical grade unless otherwise specified. The whole experiment uses deionized water with an impedance of $>18 \mathrm{M} \mathrm{cm}$.

Place $\mathrm{FeCl} 36 \mathrm{H} 2 \mathrm{O}$ in a four-reaction flask system, measure $100 \mathrm{~mL}$ of three distilled water, add $\mathrm{FeCl} 36 \mathrm{H} 2 \mathrm{O}$ to the reaction flask, protect with nitrogen, and add $\mathrm{FeCl} 2 \cdot 4 \mathrm{H} 2 \mathrm{O}$ while stirring vigorously. The inlet is added dropwise to the reaction system. When the $\mathrm{pH}$ of the solution rises to about 9.0, stop adding ammonia. Continue the reaction for $15 \mathrm{~min}$. Heat to $80^{\circ} \mathrm{C}$ and mature for $30 \mathrm{~min}$.

Preparation of nano-Fe3O4@Au. Take $20 \mathrm{~mL}$ of Fe3O4 solution for magnetic separation and wash it with sodium citrate solution $(0.2 \mathrm{~g}$ sodium citrate in $100 \mathrm{~mL}$ of trihydrated water) for 3 times, and finally dissolve in $100 \mathrm{~mL}$ of the same concentration of citric acid. In the sodium solution, placed in an ultrasonic machine for several hours of ultrasound. Place the mixture after sonication in a flask and slowly stir while heating to $70^{\circ} \mathrm{C}$. Slowly add $\mathrm{HAuCl} 4$ solution while stirring vigorously, and stop heating after $1 \mathrm{~h}$ of reaction. Stir for $40 \mathrm{~min}$. Stir the reaction.

Coupling of Integrin v3 with FITC. Add $0.1 \mathrm{mg} / \mathrm{mL}$ FITC in DMSO to $400 \mathrm{~L}$ Integrin v3 monoclonal antibody in PBS. Mix well and protect from light at $4{ }^{\circ} \mathrm{C}$ in the refrigerator. After several hours, the reaction-forming Integrin v3 mAb-FITC was dialyzed several times with a $1 \mathrm{kDa}$ semipermeable membrane (Millipore) until all unreacted free FITC was removed. 
Functional modification of nano-Fe3O4@Au. Add 100L of $1 \mathrm{mg} / \mathrm{mL} \mathrm{mPEG}-\mathrm{SH}$ to $1 \mathrm{~mL}$ of nano-Fe3O4@ Au solution. After ultrasonic reaction for 30 min, add 100L 11-MUA solution to continue the number of ultrasonic reactions. Hour. Magnetic separation wash to remove unattached reagent molecules, then add EDC and NHS, and FITCincorporated Integrin v3 monoclonal antibody. After $4{ }^{\circ} \mathrm{C}$ overnight in the dark, the magnetic separation is washed several times. Remove excess reagents that are not connected.

U87-MG human glioma cells and MCF-7 human breast cancer cells were cultured in EMEM medium and DMEM medium respectively. $10 \%$ fetal bovine serum and $1 \%$ penicillin streptomycin were added to the culture medium. Conditions are set to $37^{\circ} \mathrm{C}$, $5 \% \mathrm{CO} 2,95 \%$ air.

Incubate 500L cells with bio-modified Fe304@Au nano-solution in an incubator for $1 \mathrm{~h}$, then gently rinse the cells with fresh medium to elute non-phagocytic nanoparticles. Blocked U87-MG cells were pre-treated The integrin v3 monoclonal antibody was blocked. The treated cells were imaged using a confocal microscope (LSM510/ConfoCor2) combined system (Zeiss, Germany). The excitation source was a $488 \mathrm{~nm}$ Ar ion laser.

\section{Results and discussion}

In order to verify the successful synthesis of Fe3O4@Au nanoparticles, we characterized Fe3O4@Au nanoparticles. From the TEM photos of Fe3O4 and Fe3O4@ Au (Fig. 1(a) and (b)), Fe3O4@Au nanometers can be seen. The radius of the particles is larger than that of $\mathrm{Fe} 3 \mathrm{O} 4$, and the dispersion of Fe3O4@Au nanoparticles is better than that of $\mathrm{Fe} 3 \mathrm{O} 4$. The main reason is that $\mathrm{Au}$ is coated on the surface of Fe3O4 nanoparticles to form Fe3O4@Au nanoparticles, and the gold shell increases the stability of $\mathrm{Fe} 3 \mathrm{O} 4$ nanoparticles. Fig. 2(a) is the absorption spectrum of Fe3O4 nanoparticles, gold nanospheres and Fe3O4@Au nanoparticles. The absorption peak of Fe3O4@Au nanoparticles is 526 nm, and the spectral characteristics are the same as those of nanogold spheres. Fig. Fe3O4@Au The absorption peak of the nanoparticles is slightly red-shifted (compared to the nano-gold sphere), probably due to the increase of the radius. In the surface Raman enhancement technique, Raman scattering can be enhanced when gold is adsorbed on the surface of the detector, and then Increase the sensitivity of Raman detection. As shown in Figure 2(b), the Raman signal of 4,4'bipyridine is very weak, but its Raman is present when nano-gold spheres or Fe304@ Au nanoparticles are present on its surface. The signal is enhanced. However, Fe3O4 nanoparticles do not have this effect. Therefore, the Raman enhancement effect of Fe304@Au nanoparticles is derived from the gold shell on the surface. The above experiments show that $\mathrm{Au}$ has been coated on the surface of $\mathrm{Fe} 3 \mathrm{O} 4$ core to form Fe304@Au nanometer. particle. 

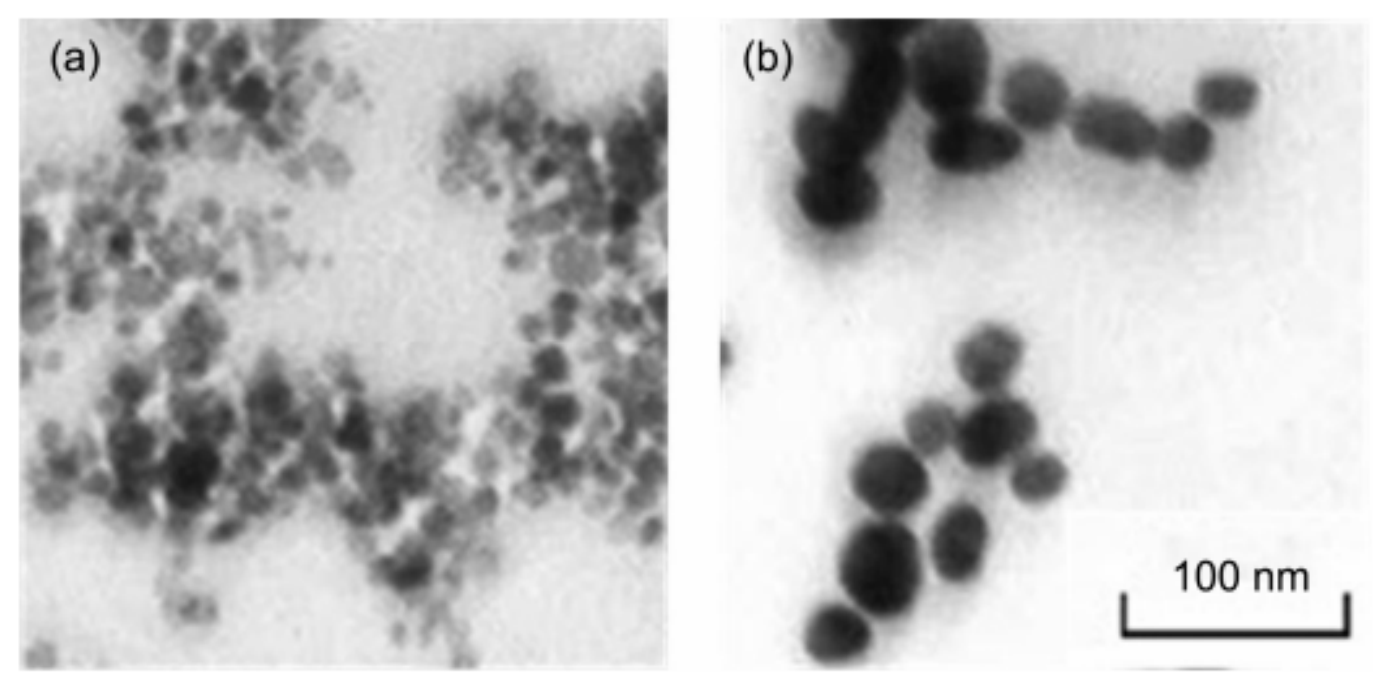

Figure 1. TEM analysis of Fe nanoparticles

Nuclear magnetic imaging is a non-destructive clinical imaging technique. In order to prove that Fe3O4@Au nanoparticles can be used as probes for nuclear magnetic imaging, the image enhancement effects of Fe3O4@Au and Fe3O4 nanoparticles were detected by nuclear magnetic T2 sequence. Figure 3(a) shows with the increase of [Fe] concentration (0, 0.1, 0.2, 0.4, 0.8, $1 \mathrm{mmol} / \mathrm{L})$, the images of Fe3O4@Au and Fe3O4 nanoparticles become darker. The enhancement effect of Fe3O4@Au nanoparticles is slightly better than that of $\mathrm{Fe} 3 \mathrm{O} 4$ nanoparticles. Poor. The transverse relaxation time of Fe3O4@Au nanoparticles is $26.53 \mathrm{~m}(\mathrm{~mol} / \mathrm{L}) 1 \mathrm{~s} 1$, and the transverse relaxation time of Fe3O4 nanoparticles is $36.67 \mathrm{~m}(\mathrm{~mol} / \mathrm{L}) 1 \mathrm{~s} 1$. Other Fe3O4/Au hybrid nanoparticles reported in the literature (such as dumbbell-shaped $\mathrm{Au}-\mathrm{Fe} 3 \mathrm{O} 4$ [13] and iron oxide/Au hybrid nanoparticles [7] connected by organic matter), Fe3O4@ at the same [Fe] concentration $\mathrm{Au}$ has similar nuclear magnetic contrast enhancement effects. In addition, due to the Au shell, the relaxation time of Fe3O4@Au nanoparticles is smaller than that of $\mathrm{Fe} 3 \mathrm{O} 4$ nanoparticles. However, some commercial T2 weight nuclear magnetic contrast agents (such as dextran package) Compared to nano-iron oxide), Fe3O4@Au nanoparticles have Good stability properties and can be modified, the stability of these properties mainly from gold shell and easily modifying. 


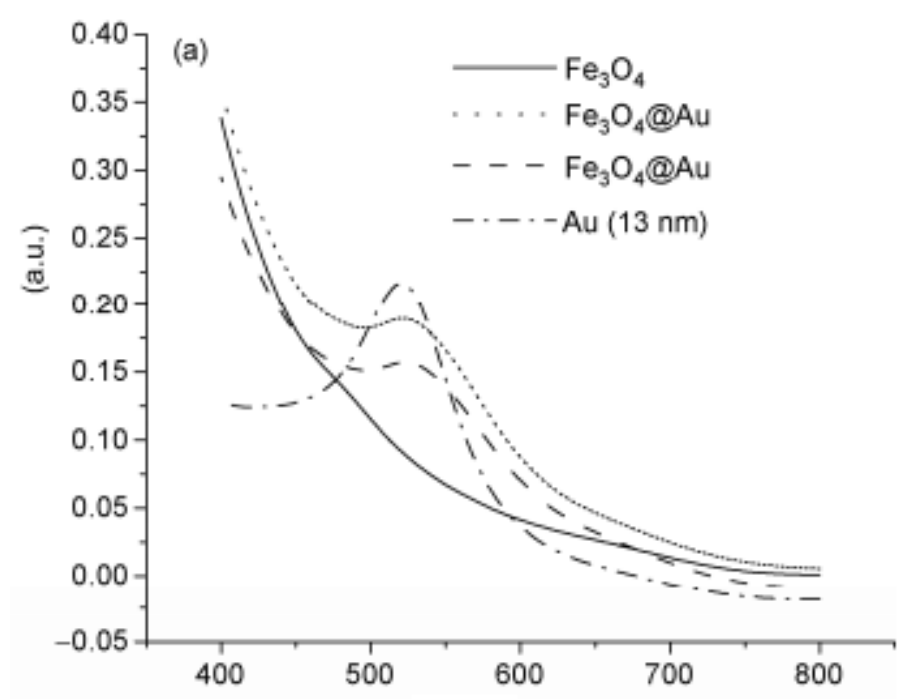

Figure 2. Releasing kinetic analysis

Photoacoustic imaging is a new type of non-destructive medical imaging technology that has just been developed in recent years. This imaging mode combines the high selectivity of optical imaging with the high resolution of ultrasound imaging [14 18]. Gold nanoparticles have been proven very It is suitable as a probe for photoacoustic imaging. In order to prove that Fe3O4@Au nanoparticles can be used as probes for photoacoustic imaging, the photoacoustic signals of Fe3O4@Au nanoparticles at different concentrations are measured. As shown in Fig. 3(b), With the increase of [Au] concentration $(0.1,0.2,0.4,0.6,0.8,1 \mathrm{mmol} / \mathrm{L})$, the photoacoustic signal of Fe3O4@Au nanoparticles is continuously enhanced, and it turns out that Fe3O4@Au nanoparticles can be used as photoacoustic imaging. Probe. The above experiments show that Fe3O4@Au nanoparticles can enhance both nuclear magnetic and photoacoustic signals, so it can be used as a dual-mode probe for nuclear magnetic and photoacoustic imaging. 

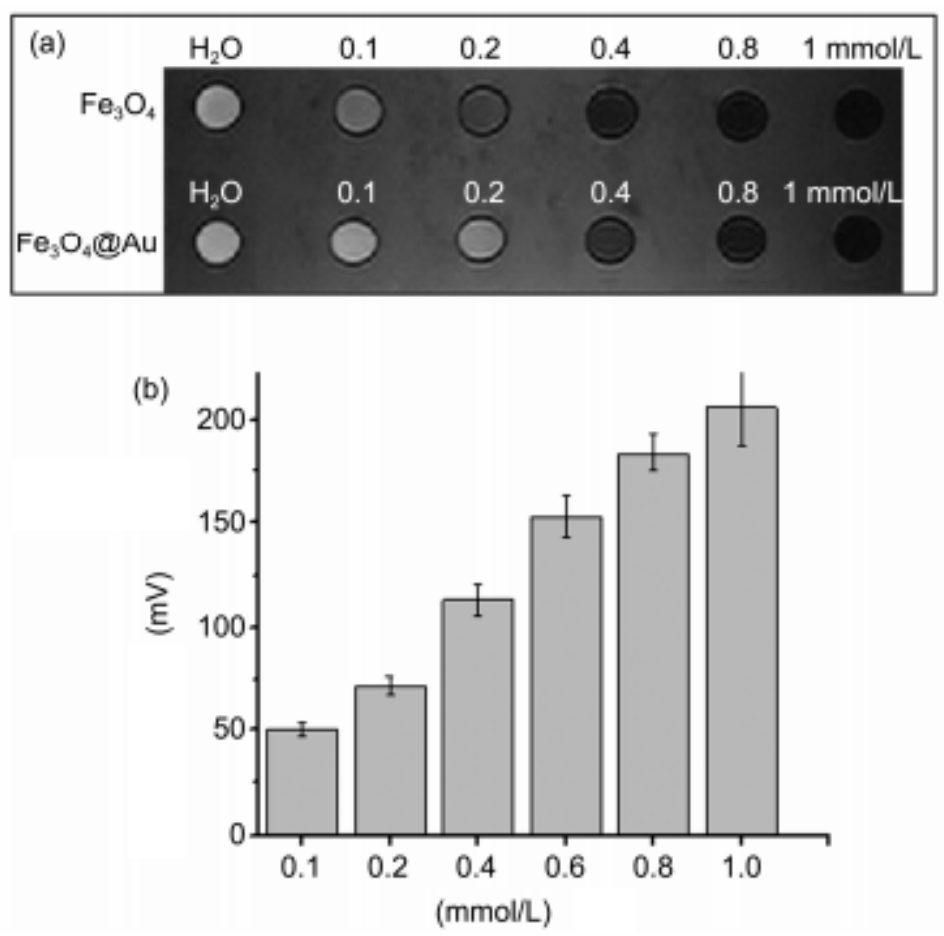

Figure 3. Cytotoxicity analysis of Fe nanoparticles

In order to make the Fe3O4@Au nanoparticles have tumor cell targeting function, we modified the Integrin v3 monoclonal antibody on the surface. At the same time, the Integrin v3 monoclonal antibody-conjugated Fe3O4@Au nanoparticle was labeled with FITC. The particles were observed by confocal microscopy to see if the nanoparticles entered the tumor cells. U87-MG tumor cells overexpressed v3 on the surface of the cell membrane. However, there were only a few v3 on the surface of MCF-7 tumor cells. Bio-modified Fe3O4@ Au nanoparticles were co-incubated with U87-MG and MCF-7, respectively. As shown in Figure 4, in the confocal microscopy images, there was a strong fluorescent signal in the cultured U87-MG tumor cells, whereas MCF-7 tumor cells No fluorescence signal was found in the U87-MG tumor cells of the control group. The confocal experiments of the cells demonstrated that the bio-modified Fe3O4@Au nanoparticles have selective targeting to U87-MG tumor cells, and the bio-modified Fe3O4@Au nanometer. Particles may be used in tumor diagnosis in the future. 


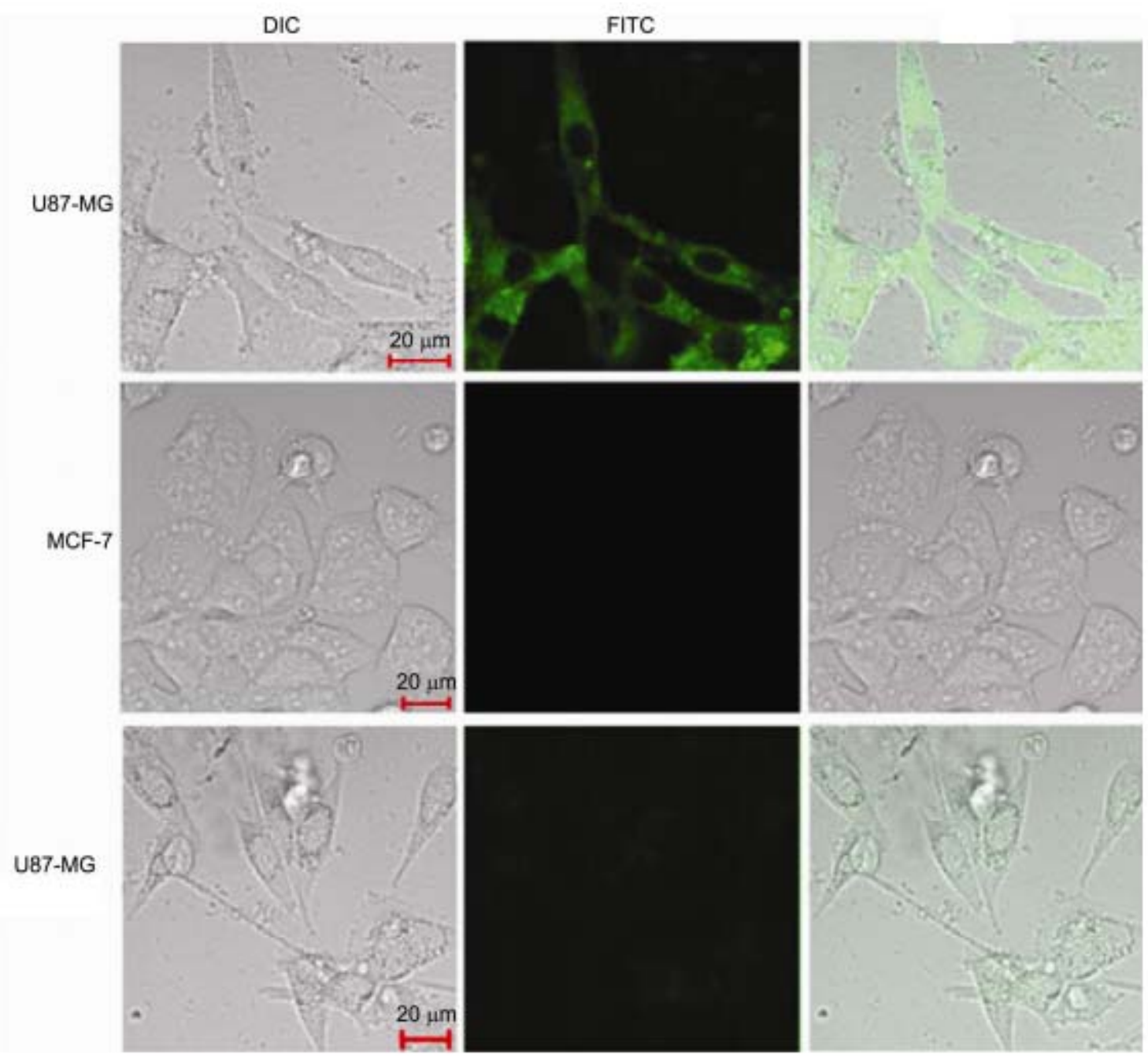

Figure 4. Cell uptake analysis by fluorescent microscopy.

Confocal fluorescence experiments demonstrated that Fe3O4@Au nanoparticles can be effectively targeted to U87-MG tumor cells. Different numbers of U87-MG tumor cells were labeled with Fe3O4@Au nanoparticles, using nuclear magnetic imaging system and photoacoustic microscopy, respectively. The imaging system images four different numbers of cell samples (approximately $1.2 \times 105,3.6 \times 106,7.4 \times 106,2.5 \times 107$ ) (Figure 5). As the number of labeled cells increases, the nuclear magnetic signal and light of the T2 sequence The acoustic signal is enhanced, and the minimum detection amount of the two imaging pairs is about $3 \times 106$. The above experiments fully prove that when the U87-MG cells are labeled by Fe3O4@Au nanoparticles, they can be simultaneously tracked by nuclear magnetic/photoacoustic imaging. In in vivo tumor diagnosis, nuclear magnetic imaging can be used as a systemic scan to determine the location of the tumor; photoacoustic imaging can analyze the physiological parameters of the tumor microenvironment, such as blood oxygen saturation, $\mathrm{pH}$. Because photoacoustic imaging has submicron level Resolution, which can obtain morphological 
information of tiny areas of the tumor (such as micro-new blood vessel structure), and then determine the nature of the tumor from a morphological point of view. The decorated Fe304@Au nanoparticles can be actively targeted to U87-MG tumor cells, and the labeled U87-MG tumor cells can be tracked by nuclear magnetic/photoacoustic imaging. The combined imaging of nuclear magnetic and photoacoustic can be multiparameter, multi-scale Multi-dimensional reflection of tumor information. Bio-modified Fe304@Au nanoparticles will greatly promote the application of nuclear magnetic and photoacoustic dual-mode imaging in clinical practice.

\section{Conclusion}

In this paper, bio-modified Fe304@Au nanoparticles were successfully synthesized. The bio-modified Fe304@Au nanoparticles can simultaneously enhance the nuclear magnetic signal and photoacoustic signal of T2 sequence. This probe has selective targeting to U87-MG tumor cells. U87- MG tumor cells after novel probe labeling can be simultaneously tracked by nuclear magnetic and photoacoustic imaging. Bio-modified Fe304@Au nanoparticles will be the bridge between nuclear magnetic and photoacoustic dual-mode imaging. Nuclear magnetic/photoacoustic dual-mode imaging will be able to obtain more Multiple tumor morphology and physiological information, nuclear magnetic/photoacoustic dual-mode imaging based on Fe3O4@Au nanoparticles will play an important role in the clinical diagnosis of tumors.

\section{References}

1. Laird, P. W.; Zijderveld, A.; Linders, K.; Rudnicki, M. A.; Jaenisch, R.; Berns, A., Simplified mammalian DNA isolation procedure. Nucleic acids research 1991, 19 (15), 4293.

2. Boussif, O.; Lezoualc'h, F.; Zanta, M. A.; Mergny, M. D.; Scherman, D.; Demeneix, B.; Behr, J.-P., A versatile vector for gene and oligonucleotide transfer into cells in culture and in vivo: polyethylenimine. Proceedings of the National Academy of Sciences 1995, 92 (16), 7297-7301.

3. Kenworthy, A. K.; Hristova, K.; Needham, D.; Mclntosh, T. J., Range and magnitude of the steric pressure between bilayers containing phospholipids with covalently attached poly (ethylene glycol). Biophysical journal 1995, 68 (5), 1921-1936.

4. Krieg, A. M.; Yi, A.-K.; Matson, S.; Waldschmidt, T. J.; Bishop, G. A.; Teasdale, R.; Koretzky, G. A.; Klinman, D. M., CpG motifs in bacterial DNA trigger direct B-cell activation. Nature 1995, 374 (6522), 546.

5. Zhang, R.; Billingsley, M. M.; Mitchell, M. J., Biomaterials for vaccine-based cancer immunotherapy. Journal of Controlled Release 2018.

6. Zhang, R.; Kramer, J. S.; Smith, J. D.; Allen, B. N.; Leeper, C. N.; Li, X.; Morton, L. D.; Gallazzi, F.; Ulery, B. D., Vaccine Adjuvant Incorporation Strategy Dictates Peptide Amphiphile Micelle Immunostimulatory Capacity. The AAPS journal 2018, 20 (4), 73. 
7. Zhang, R.; Leeper, C. N.; Wang, X.; White, T. A.; Ulery, B. D., Immunomodulatory vasoactive intestinal peptide amphiphile micelles. Biomaterials science 2018.

8. Zhang, R.; Leeper, C. N.; Wang, X.; White, T. A.; Ulery, B. D., Immunomodulatory vasoactive intestinal peptide amphiphile micelles. Biomaterials science 2018, 6 (7), 1717-1722.

9. Zhang, R.; Morton, L. D.; Smith, J. D.; Gallazzi, F.; White, T. A.; Ulery, B. D., Instructive Design of Triblock Peptide Amphiphiles for Structurally Complex Micelle Fabrication. ACS Biomaterials Science \& Engineering 2018.

10. Zhang, R.; Smith, J. D.; Allen, B. N.; Kramer, J. S.; Schauflinger, M.; Ulery, B. D., Peptide Amphiphile Micelle Vaccine Size and Charge Influence the Host Antibody Response. ACS Biomaterials Science \& Engineering 2018.

11. Zhang, R.; Smith, J. D.; Allen, B. N.; Kramer, J. S.; Schauflinger, M.; Ulery, B. D., Peptide Amphiphile Micelle Vaccine Size and Charge Influence the Host Antibody Response. ACS Biomaterials Science \& Engineering 2018, 4 (7), 2463-2472.

12. Zhang, R.; Ulery, B. D., Synthetic vaccine characterization and design. Journal of Bionanoscience 2018, 12 (1), 1-11.

13. Smith, J. D.; Cardwell, L. N.; Porciani, D.; Nguyen, J. A.; Zhang, R.; Gallazzi, F.; Tata, R. R.; Burke, D. H.; Daniels, M. A.; Ulery, B. D., Aptamer-displaying peptide amphiphile micelles as a cell-targeted delivery vehicle of peptide cargoes. Physical biology 2018, 15 (6), 065006.

14. Pack, D. W.; Hoffman, A. S.; Pun, S.; Stayton, P. S., Design and development of polymers for gene delivery. Nature reviews Drug discovery 2005, 4 (7), 581.

15. Torchilin, V. P., Recent advances with liposomes as pharmaceutical carriers. Nature reviews Drug discovery 2005, 4 (2), 145.

16. Minigo, G.; Scholzen, A.; Tang, C. K.; Hanley, J. C.; Kalkanidis, M.; Pietersz, G. A.; Apostolopoulos, V.; Plebanski, M., Poly-L-lysine-coated nanoparticles: a potent delivery system to enhance DNA vaccine efficacy. Vaccine 2007, 25 (7), 1316-1327.

17. Akinc, A.; Zumbuehl, A.; Goldberg, M.; Leshchiner, E. S.; Busini, V.; Hossain, N.; Bacallado, S. A.; Nguyen, D. N.; Fuller, J.; Alvarez, R., A combinatorial library of lipidlike materials for delivery of RNAi therapeutics. Nature biotechnology 2008, 26 (5), 561.

18. Bowie, A. G.; Unterholzner, L., Viral evasion and subversion of patternrecognition receptor signalling. Nature Reviews Immunology 2008, 8 (12), 911.

19. Weide, B.; Carralot, J.-P.; Reese, A.; Scheel, B.; Eigentler, T. K.; Hoerr, I.; Rammensee, H.-G.; Garbe, C.; Pascolo, S., Results of the first phase I/ll clinical vaccination trial with direct injection of mRNA. Journal of immunotherapy 2008, 31 (2), 180-188.

20. Chen, D.; Love, K. T.; Chen, Y.; Eltoukhy, A. A.; Kastrup, C.; Sahay, G.; Jeon, A.; Dong, Y.; Whitehead, K. A.; Anderson, D. G., Rapid discovery of potent siRNAcontaining lipid nanoparticles enabled by controlled microfluidic formulation. Journal of the American Chemical Society 2012, 134 (16), 6948-6951.

21. Fields, R. J.; Cheng, C. J.; Quijano, E.; Weller, C.; Kristofik, N.; Duong, N.; Hoimes, C.; Egan, M. E.; Saltzman, W. M., Surface modified poly ( $\beta$ amino ester)containing nanoparticles for plasmid DNA delivery. Journal of controlled release 2012, $164(1), 41-48$. 
22. Leung, A. K.; Hafez, I. M.; Baoukina, S.; Belliveau, N. M.; Zhigaltsev, I. V.; Afshinmanesh, E.; Tieleman, D. P.; Hansen, C. L.; Hope, M. J.; Cullis, P. R., Lipid nanoparticles containing siRNA synthesized by microfluidic mixing exhibit an electrondense nanostructured core. The Journal of Physical Chemistry C 2012, 116 (34), 18440-18450.

23. Allen, T. M.; Cullis, P. R., Liposomal drug delivery systems: from concept to clinical applications. Advanced drug delivery reviews 2013, 65 (1), 36-48.

24. Mui, B. L.; Tam, Y. K.; Jayaraman, M.; Ansell, S. M.; Du, X.; Tam, Y. Y. C.; Lin, P. J.; Chen, S.; Narayanannair, J. K.; Rajeev, K. G., Influence of polyethylene glycol lipid desorption rates on pharmacokinetics and pharmacodynamics of siRNA lipid nanoparticles. Molecular Therapy-Nucleic Acids 2013, 2.

25. Mui, B. L.; Tam, Y. K.; Jayaraman, M.; Ansell, S. M.; Du, X.; Tam, Y. Y. C.; Lin, P. J.; Chen, S.; Narayanannair, J. K.; Rajeev, K. G., Influence of polyethylene glycol lipid desorption rates on pharmacokinetics and pharmacodynamics of siRNA lipid nanoparticles. Molecular Therapy-Nucleic Acids 2013, 2, e139. 